\section{AB0534 THROMBOTIC MICROANGIOPATHY IN PREGNANT WOMEN WITH SYSTEMIC LUPUS ERYTHEMATOSUS: CHARACTERICTICS AND OUTCOMES}

T. Kirsanova ${ }^{1}$, M. Vinogradova ${ }^{1}$, A. Kolyvanova ${ }^{2}$, N. Klimenchenko ${ }^{1} .{ }^{1}$ Federal State Budget Institution "Research Center for Obstetrics, Gynecology and Perinatology" MoH, Russian Federation; ${ }^{2}$ Moscow State University, Moscow, Russian Federation

Objectives: to evaluate the impact of thrombotic microangiopathy (TMA) on perinatal and maternal outcomes in patients with systemic lupus erythematosus (SLE).

Methods: This study included 21 SLE patients with pregnancy, who developed TMA signs during pregnancy. Patients underwent general survey, clinical and laboratory assessment for disease activity and APL-antibodies. All of them had low SLE-activity indices.

Results:

Table 1. Laboratory features of pregnant patients with SLE

\begin{tabular}{lcc}
\hline & TMA $(\mathrm{n}=7)$ & Without TMA $(\mathrm{n}=14)$ \\
\hline $\mathrm{Hb}, \mathrm{g} / \mathrm{l}$ & 89,8 & 95,8 \\
LDH, Ed/l & 716,39 & 331,2 \\
ALT, Ed/l & 110,9 & 21,3 \\
AST,Ed/l & 69,1 & 24,3 \\
Creatinine, mkmol/l & 113,3 & 77,3 \\
GFR, $\mathrm{ml} / \mathrm{min}$ & 50,5 & 81,1 \\
Proteinuria, g/24h & 0,65 & 0,28 \\
Thrombocytes, $\times 10^{9}$ & 135,5 & 213,8 \\
sFLT/PLGF & 66,6 & 18,9 \\
\hline
\end{tabular}

Table 2. Perinatal outcomes in pregnant women with SLE

\begin{tabular}{lcc}
\hline & TMA $(n=7)$ & Without TMA $(n=14)$ \\
\hline - Livebirth & 4 & 12 \\
- Stillbirth & 3 & 1 \\
Weigh & 1363,3 & 3047,3 \\
Mean gestational age & 29,1 & 36,2 \\
\hline
\end{tabular}

TMA signs were found in $7 / 21$ SLE patients (33\%). Patients with TMA had significantly higher systolic and diastolic blood pressure $(P \leq 0.05)$, higher serum creatinine and lower estimated glomerular filtration rate $(P \leq 0.05)$ than that of those without TMA lesions (tabl.1). APLA positivity was significantly more frequent in patients with TMA (3/7 vs 0/14). TMA onset in 2 patients occurred before 20 gestational weeks, 5/7 have developed PE signs (23-38 weeks), only 3/7 had classical HELLP syndrome. There was no PE in patients without TMA. There was a significant association between the detection of TMA and adverse perinatal outcomes (tabl.2).

Patient with TMA and SLE had a poor outcome and most severe course: 5/7 had a PE (3/7 developed HELLP),3/7 had antenatal fetal death, 3/7had signs of heart damage, $5 / 7$ a variety of neurological manifestations. Despite of PE signs in 5/7 with TMA the blood sFlt1/PLGF levels were slightly above normal (average sFlt/PLGF levels in preeclampsia without SLE in our another study is 404,47 )

Conclusions: TMA is not an uncommon disorder in pregnant patients with SLE. It is associated with APLA positivity only in $1 / 3$ cases. TMA was significantly associated with renal impairment, systemic hypertension and adverse perinatal outcomes. Less increased ratio of sFIt-1/PIGF in TMA patients than "pure" PE may confirm that PE in patients with TMA is not a condition due to angiogenic factors imbalance. Circulating concentrations of angiogenic factors appear not to be suitable markers to assess the severity of PE and adverse outcomes in SLE patients. Thus, TMA may be an important cause of renal injury and renal dysfunction in a subset of pregnant patients with SLE and associated with worse renal and perinatal prognosis. Complement over-activation via both classical (SLE+APS) and alternative pathways might play an important role in the pathogenesis of TMA in SLE.

Disclosure of Interest: None declared

DOI: 10.1136/annrheumdis-2017-eular.6589

\section{AB0535 FATIGUE IS ASSOCIATED WITH HS-CRP IN PATIENTS WITH SYSTEMIC LUPUS ERYTHEMATOSUS}

T. Aung, L. Sahakian, B. Skaggs, J. Grossman, M. McMahon. Rheumatology, UCLA, Los Angeles, California, United States

Background: Fatigue is highly prevalent and has a negative impact on quality of life in patients with Systemic Lupus Erythematosus. ${ }^{1,2}$ The 9-item Fatigue Severity Scale (FSS) is one of the commonly used self-report questionnaires to measure fatigue in patients with chronic disease. ${ }^{3}$ Although fatigue is one of the most commonly reported symptoms in SLE, previous studies have not consistently found any clear association between disease activity (as measured by the SLEDAI) ${ }^{4}$ or with laboratory measures of disease activity. ${ }^{1}$

Objectives: To determine whether fatigue is associated with any laboratory measures of disease activity or inflammation in patients with SLE.

Methods: SLE patients and age-matched controls participating in our longitudinal "Biomarkers of Cardiovascular Disease in SLE" completed FSS questionnaires at study entry. Laboratory measures of cardiovascular risk assessment and lupus disease activity assessments were performed in plasma, including high sensitivity C-reactive protein (hs-CRP), myeloperoxidase (MPO), homocysteine, fasting lipid panels, erythrocyte sedimentation rate (ESR), C3, C4, dsDNA, and urine protein/creatinine ratio (UPCR).

Results: 54 patients and 37 control individuals participated. FSS scores were higher in patients with SLE $(4.79 \pm 1.63$ (mean $\pm \mathrm{SD}$ ) compared to healthy subjects $(3.17 \pm 1.83, \mathrm{P}<0.001)$. There were no significant correlations between FSS and age, gender, or SLE disease duration.

In SLE patients, there was significant correlation between FSS scores and hs$\operatorname{CRP}(r=0.56, p=0.004)$, and an inverse correlation with myeloperoxidase $(-0.37$, $\mathrm{p}=0.045$ ). No associations between FSS and C3, C4, dsDNA, ESR, UPCR, fasting lipid levels, or homocysteine were seen in the SLE group. No associations between FSS and laboratory measures of cardiovascular risk assessment were seen in the control group.

Conclusions: Although fatigue is not correlated with traditional laboratory measures of disease activity in SLE patients, we did find significant associations between fatigue, hs-CRP, and myeloperoxidase in our cohort. Future longitudinal studies are underway to determine whether these associations also reflect the risk for progression of cardiovascular disease in patients with high fatigue. References:

[1] Krupp L B, LaRocca N G MJ et al. A study of fatigue in systemic lupus erythematosus. J Rheumatol. 1990;17(11):1450-1452.

[2] Tench CM, McCurdie I, White PD, D'Cruz DP. The prevalence and associations of fatigue in systemic lupus erythematosus. Rheumatology (Oxford). 2000;39(11):1249-1254. doi:10.1093/rheumatology/39.11.1249.

[3] Hjollund NH, Andersen JH, Bech P. Assessment of fatigue in chronic disease: a bibliographic study of fatigue measurement scales. Health Qual Life Outcomes. 2007;5:12. doi:10.1186/1477-7525-5-

[4] Wang B, Gladman DD, Urowitz MB. Fatigue in lupus is not correlated with disease activity. J Rheumatol. 1998;25(5):892-895.

Disclosure of Interest: None declared

DOI: 10.1136/annrheumdis-2017-eular.2175

\section{AB0536 ANTICARDIOLIPIN ANTIBODIES IN SYSTEMIC LUPUS ERYTHEMATOSUS}

T. Ben Salem, I. Naceur, M. Tougorti, M. Lamloum, I. Ben Ghorbel,

M.H. Houman. Internal Medicine, Rabta university hospital, Tunis, Tunisia

Background: Anticardiolipin (ACL) antibodies have been independently associated with high incidence of thrombotic diseases and pregnancy associated disorders in patients with systemic lupus erythematosus (SLE).

Objectives: This study aims to assess influence of ACL antibodies in SLE and their association with clinical, biological and immunological features.

Methods: A retrospective study of 246 patients with systemic lupus erythematous (revised ACR criteria) conduced in an internal medicine department over a period time of 14 years. Two groups were compared according to the positivity of ACL antibodies (group1: positive ACL/ group2: negative ACL).

Results: ACL antibodies were positive in $48.8 \%$ of SLE patients (screened in 168 patients). This prevalence was similar in female and male. Poor general state at diagnosis was more frequent in the ACL positive group ( $55.7 \%$ vs $38.6 \%$; $p=0.02$ ). The following data were comparable in the two groups: joint involvement $(91.8 \%$ vs $90.8 \% ; \mathrm{p}=0.7)$, pericarditis $(33.8 \%$ vs $37.8 \% ; \mathrm{p}=0.6)$ and pleural effusion (24.1\% vs $29.5 \%$; $p=0.44$ ). Alopecia ( $38.6 \%$ vs $27.9 \% ; p=0.2$ ), central nervous system involvement $(12.5 \%$ vs $18.8 \% ; p=)$ and peripheral neuropathy $(6.4 \%$ vs $11.1 \%$; $\mathrm{p}=$ ) were more frequent in the group 2 without significant difference. Prevalence of lupus nephritis was more important in the group $1(52.8 \%$ vs $38.8 \% ; \mathrm{p}=0.07)$. Thromboembolic complications: deep venous thrombosis $(13.8 \%$ vs $11.9 \% ; p=0.72)$ and pulmonary embolism ( $10.8 \%$ vs $7.1 \% ; p=0.51)$ were more frequent in patients with ACL antibodies without significant difference. Obstetrical complications were more frequent in group 1 but differences were not statistically significant; spontaneous abortions $(29.5 \%$ vs $20.4 \% ; p=0.29)$, intra-uterin death (13.6\% vs $9.3 \%$; $p=0.5)$, premature birth $(6.8 \%$ vs $1.9 \%$; $p=0.3$ ).

There were no associations between hematologic disorders and ACL antibodies; leucopenia ( $51.3 \%$ vs $39.5 \% ; p=0.13$ ) and), lymphopenia ( $81 \%$ vs $80.2 \% ; p=0.9)$, anemia $(77.2 \%$ vs $74.4 ; p=0.6)$ and thrombopenia $(22.8 \%$ vs $23.5 \% ; p=0.9)$ were comparable in the two groups. Frequencies of anti-ds DNA antibody and of antibodies to extractable nuclear antigens were similar in the two groups. Only anti-B2GP1 antibodies were significantly associated to ACL antibodies $(51.3 \%$ vs $17.2 \% ; p<0.001$.

Conclusions: ACL antibodies were found in $30-40 \%$ of patients with SLE, this is comparable to our group.

No significant associations of $\mathrm{ACL}$ antibodies.to thrombotic events or obstetrical complications were found in our patients

Disclosure of Interest: None declared

DOI: 10.1136/annrheumdis-2017-eular.3909 